\title{
Design of Robust Systems for Stabilization of Unmanned Aerial Vehicle Equipment
}

\author{
Olha Sushchenko ${ }^{1}$ and Andriy Goncharenko ${ }^{2}$ \\ ${ }^{1}$ Aircraft Control Systems Department, Educational and Research Institute of Air Navigation, National Aviation University, \\ Cosmonaut Komarova Avenue 1, Kyiv 03058, Ukraine \\ ${ }^{2}$ Aircraft Airworthiness Department, Aircraft Faculty, Educational and Research Airspace Institute, National Aviation University, \\ Cosmonaut Komarova Avenue 1, Kyiv 03058, Ukraine
}

Correspondence should be addressed to Olha Sushchenko; sushoa@ukr.net

Received 28 March 2016; Revised 1 July 2016; Accepted 17 July 2016

Academic Editor: Paul Williams

Copyright ( 2016 O. Sushchenko and A. Goncharenko. This is an open access article distributed under the Creative Commons Attribution License, which permits unrestricted use, distribution, and reproduction in any medium, provided the original work is properly cited.

\begin{abstract}
The paper deals with the structural synthesis of robust system for stabilization of observation equipment operated on unmanned aerial vehicles. The model of the triaxial stabilization system taking into consideration necessary kinematic transformations is developed. The matrix weighting transfer functions ensuring design of the system with the desired amplitude-frequency characteristics of the system are chosen. The features of the robust structural synthesis for the researched system are considered. The structure and parameters of the robust controller, based on robust structural synthesis including the methods of the mixed sensitivity and loop-shaping, are obtained. The results of the synthesized system simulation are represented. The obtained results allow implementing stabilization of observation equipment in difficult conditions of real operation. This improves the quality of photography, mapping, survey, and so forth and gives advantages of accuracy for images representations of the territory flown. The obtained results are significant for stabilization of equipment operated at a moving base.
\end{abstract}

\section{Introduction}

Nowadays unmanned aerial vehicles (UAVs) carry out different functions including photography, mapping, monitoring, and survey. Cameras, laser scanners, and other information and measuring devices can be used for solving these important tasks. The problems of cartography can be solved by means of the digital photogrammetry and aerial survey using the high resolution TV cameras. A laser scanner can be used for aerial survey. The high quality of image could be provided by means of stabilization of the information and measuring devices in the inertial space.

All these applications require solving such problems as stabilization and control by orientation of the device line-ofsight. The appropriate control systems may differ according to the performed tasks, but main functions of these systems are stabilization and pointing of the above listed devices. The main components of the systems are the platform with the payload (observation equipment), inertial measuring instruments (rate gyros, accelerometers), gimbals, and drives [1-4].

As a rule, such systems implement stabilization by two axes [2]. In the most responsible cases, it is necessary to use three-axial stabilization. The paper proposes a method of design of the robust system providing stabilization of UAV payload by three axes. Such choice improves functionality of the system. In this case, it is possible both to stabilize platform and to control by platform angular position providing the necessary orientation of the payload line-of-sight.

Control in conditions of uncertainty is very important for design problems of the modern UAVs. The robust stabilization provides the required quality of operation in conditions of changed plant parameters and external disturbances. It is worth noticing that implementation of the robust systems is less expensive in comparison with the adaptive systems. Therefore the robust systems have some advantages in comparison with adaptive ones. 
The paper deals with design of a robust system of the researched type. Such system provides the robust stability and robust performance in conditions of parametrical and coordinate (external) disturbance. It is based on minimization of $H_{\infty}$-norm of the system sensitivity and complementary sensitivity functions.

Operation of the observation equipment at UAV is implemented in conditions of the external disturbances caused, first of all, by wind influence. Required accuracy of stabilization, tracking, and pointing in such conditions could be achieved by means of the robust control.

Features of the robust control systems design are represented in many papers and textbooks [5-8]. It should be noticed that the modern scientific-technical literature gives sufficient attention to the design of the robust systems for control of aircrafts motion $[9,10]$. At the same time problems of creation of the robust systems for stabilization of equipment operated at aircrafts have no appropriate development. The method described in the paper allows solving these problems. Papers $[11,12]$ are dedicated to the applicable issues. Some basic concepts and representations of the considered approach are briefly given in [12].

The paper deals with the robust stabilization of the observation equipment operated at UAV. This is a new problem in the framework of the robust systems application.

Such approach provides the needed accuracy of stabilization in difficult conditions of UAV real operation. At that, costs for design works are much lower than those for adaptive systems. The lower cost of the design works is an advantage of the system of the researched type.

Expediency of alternative aircraft or their equipment type use can be modeled in the paradigm described and discussed in $[13]$.

The paper proposes mathematical model of the stabilization plant and the weighting transfer functions for the plant augmentation and represents features of the robust structural synthesis for the specified systems.

\section{Mathematical Model of Stabilization Plant}

A stabilization plant represents a platform with observation equipment and measuring instruments. In order to provide the high accuracy of the observation processes, stabilization must be implemented by all three UAV axes. The stabilized platform is installed into gimbals with three degrees of freedom. Such approach makes it possible to implement the platform rotations in a sufficiently wide range of angles such as 360 degrees of the yaw and \pm 90 degrees of angles of the pitch and roll. (In practical situations rotations at angles of the pitch and the roll are bounded by values \pm 70 degrees.) In the general case, stabilization system may operate in modes of prestabilization and precision stabilization. Control in the prestabilization mode may be realized by means of accelerometers (in fact deviation from stabilization plane may be determined using accelerometers signals because $\alpha=w / g$; here $\alpha$ is angle of a platform deviation, $w$ is acceleration, and $g$ is the gravity acceleration).

These modes require using of the inertial sensors such as accelerometers and rate gyros. The pendulous accelerometers and MEMS-gyros can be used as measuring instruments for systems of the researched kind.

A mathematical model of such a plant must include equations of dynamics and kinematics. The dynamics of the platform could be described by Euler equations [14]:

$$
\begin{aligned}
& \dot{\omega}_{X} J_{X}+\omega_{Y} \omega_{Z}\left(J_{Z}-J_{Y}\right)-\left(\omega_{Y}^{2}-\omega_{Z}^{2}\right) J_{Y Z} \\
& \quad-\left(\omega_{X} \omega_{Y}+\dot{\omega}_{Z}\right) J_{X Z}+\left(\omega_{X} \omega_{Z}-\dot{\omega}_{Y}\right) J_{X Y}=M_{X} ; \\
& \dot{\omega}_{Y} J_{Y}+\omega_{X} \omega_{Z}\left(J_{X}-J_{Z}\right)-\left(\omega_{Z}^{2}-\omega_{X}^{2}\right) J_{X Z} \\
& \quad-\left(\omega_{Z} \omega_{Y}+\dot{\omega}_{X}\right) J_{X Y}+\left(\omega_{X} \omega_{Y}-\dot{\omega}_{Z}\right) J_{Y Z}=M_{Y} ; \\
& \dot{\omega}_{Z} J_{Z}+\omega_{X} \omega_{Y}\left(J_{Y}-J_{X}\right)-\left(\omega_{X}^{2}-\omega_{Y}^{2}\right) J_{X Y} \\
& \quad-\left(\omega_{X} \omega_{Z}+\dot{\omega}_{Y}\right) J_{Y Z}+\left(\omega_{X} \omega_{Z}-\dot{\omega}_{X}\right) J_{X Z}=M_{Z}
\end{aligned}
$$

where $\omega_{X}, \omega_{Y}, \omega_{Z}$ represent projections of the angular rates on the platform axes; $J_{X}, J_{Y}, J_{Z}$ represent inertia moments of the platform with the payload and the measuring instruments relative to the gimbals axes; $J_{Y Z}, J_{X Z}, J_{X Y}$ represent the centrifugal moments of inertia relative to the gimbals axes; $\dot{\omega}_{X}, \dot{\omega}_{Y}, \dot{\omega}_{Z}$ represent projections of angular accelerations on the platform axes; $M_{X}, M_{Y}, M_{Z}$ are moments acting along the gimbals axes. It should be noted that constituents of expressions (1) $J_{X}, J_{Y}, J_{Z}$ represent the equivalent inertia moments, which include the inertia moments of the platform $J_{p}$ and the motors $J_{m}$. They can be described in the following way:

$$
J_{i}=J_{p i}+n_{r}^{2} J_{m}, \quad i=X, Y, Z,
$$

where $J_{p i}, J_{m}$ represent the inertia moments of the platform with the payload and measuring instruments and the inertia moment of the motor, respectively; $n_{r}$ represents a ratio of the reduction gear. Relationships for the centrifugal inertia moments of the platform can be derived in a similar way.

The moments acting on the platform include such components as the moments of the dry friction in bearings of the gimbals, moments created with motors, and disturbing moments.

For the system, working in the precision stabilization mode, it is necessary to take into consideration that every contour channel includes the platform with payload, motor, pulse-width-modulator (PWM), and rate gyro [12, 15]. Measuring range, sensitivity, and resistance to shocks are main characteristics of the rate gyro choice. Bandwidth of the chosen gyro is $100 \mathrm{~Hz}$. Angular random walk of the gyro is $0,3 \mathrm{deg} / \sqrt{h}$. Stability of the scale factor is of great importance for the considered application.

Components of moments acting by the gimbals axes can be defined in the following way [16]:

$$
\begin{aligned}
& M_{1 i}=M_{\mathrm{fr}} \operatorname{sign} \omega_{i} ; \\
& M_{2 i}=\frac{\widetilde{n}_{m} U_{a i}}{R_{a}} ; \\
& M_{3 i}=M_{\text {dist } i}
\end{aligned}
$$


where $M_{\mathrm{fr}}$ represents nominal moment of the dry friction in bearings mounted in the gimbals axes; $\widetilde{n}_{m}$ represents coefficient of loading at the motor shaft; $U_{a i}$ represent voltages of the motor control windings; $R_{a}$ represents resistance of the motor armature winding; $M_{\text {dist } i}$ represent disturbance moments.

The voltages created in the motor armature control windings are described by the following expressions [16, 17]:

$$
T_{a} \dot{U}_{a i}+U_{a i}=k_{\mathrm{PWM}} U_{\mathrm{PWM} i}-n_{r} c_{e} \omega_{i}, \quad i=X, Y, Z,
$$

where $T_{a}$ represents the time constant of the armature circuit; $\tilde{n}_{e}$ represents the coefficient of proportionality between the electromotive force and the angular rate of the motor; $k_{\mathrm{PWM}}$ represents the transfer constant of the linearized PWM; $U_{\mathrm{PWM} i}$ represent voltages at PWM input.

Control voltages at the output of the angular rate gyro sensors $U_{\omega i}$ can be described in the following way $[12,15]$ :

$$
T_{g}^{2} \ddot{U}_{\omega i}+2 \xi T_{g} \dot{U}_{\omega i}+U_{\omega i}=k_{g} \omega_{i}, \quad i=X, Y, Z,
$$

where $T_{g}$ represents the time constant of the rate gyro; $\xi$ represents the damping coefficient; $k_{g}$ represents the gain of the rate gyro.

In order to describe kinematics of the stabilization system of the researched kind it is necessary to take into consideration the reference frames describing a position of the stabilized platform in the inertial space.

The position of the body-axes reference frame of the platform $O X Y Z$ in the inertial space relative to the initial

$$
\begin{aligned}
& X \\
& A=\begin{array}{cc}
X_{A} & \cos \psi \cos \gamma+\sin \psi \sin \vartheta \sin \gamma \\
Y_{A} & \cos \vartheta \sin \gamma
\end{array} \\
& Z_{A}-\sin \psi \cos \gamma+\cos \psi \sin \vartheta \sin \gamma \\
& X_{A} \\
& A^{T}=\begin{array}{l}
X \cos \psi \cos \gamma+\sin \psi \sin \vartheta \sin \gamma \\
Y-\cos \psi \sin \gamma+\sin \psi \sin \vartheta \cos \gamma
\end{array} \\
& Z \quad \sin \psi \cos \vartheta
\end{aligned}
$$

\section{Y}

Determination of the stabilization angular rates can be implemented in the following way. If $\omega$ represents an angular rate of a platform, on which observation equipment and instruments for measurement of the platform angular rate in the inertial space are mounted, and $\Omega$ represents the platform angular rate conditioned by control moments, the condition of the precision stabilization becomes [18]

$$
\Omega+\omega=0 .
$$

If transition to a new position of the platform in the inertial space is implemented by means of rotations on angles $\psi$, $\vartheta, \gamma$ in accordance with Figure 1, angular rates of the gimbals reference frame $O X_{A} Y_{A} Z_{A}$ is defined by means of the sequence of rotations on angles $\psi, \vartheta, \gamma$ as it is shown in Figure 1.

The appropriate matrices of the direction cosines are represented in the form

$$
\begin{gathered}
A_{1}=\begin{array}{cccc} 
& X_{1} & Y_{1} & Z_{1} \\
X_{A} & \cos \psi & 0 & \sin \psi \\
Y_{A} & 0 & 1 & 0 \\
Z_{A} & -\sin \psi & 0 & \cos \psi \\
A_{2}= & X_{2} & Y_{2} & Z_{2} \\
X_{1} & 1 & 0 & 0 \\
Y_{1} & 0 & \cos \vartheta & -\sin \vartheta \\
Z_{1} & 0 & \sin \vartheta & \cos \vartheta \\
A_{3}= & X & Y & Z \\
X_{2} & \cos \gamma & -\sin \gamma & 0 \\
Y_{2} & \sin \gamma & \cos \gamma & 0 \\
Z_{2} & 0 & 0 & 1
\end{array} .
\end{gathered}
$$

Based on relationships (6) the matrices of direction cosines between the axes $X Y Z, X_{A} Y_{A} Z_{A}$ and the axes $X_{A} Y_{A} Z_{A}, X Y Z$ will look like

axes, taking into consideration matrices of the directional cosines (6), will be determined by the relationships [12]

$$
\begin{aligned}
& {\left[\begin{array}{l}
\omega_{X_{1}} \\
\omega_{Y_{1}} \\
\omega_{Z_{1}}
\end{array}\right]=A_{1}^{T}\left[\begin{array}{l}
\omega_{X_{A}} \\
\omega_{Y_{A}} \\
\omega_{Z_{A}}
\end{array}\right]+A_{1}^{T}\left[\begin{array}{l}
0 \\
\dot{\psi} \\
0
\end{array}\right] ;} \\
& {\left[\begin{array}{l}
\omega_{X_{2}} \\
\omega_{Y_{2}} \\
\omega_{Z_{2}}
\end{array}\right]=A_{2}^{T} A_{1}^{T}\left[\begin{array}{l}
\omega_{X_{A}} \\
\omega_{Y_{A}} \\
\omega_{Z_{A}}
\end{array}\right]+A_{2}^{T}}
\end{aligned}
$$




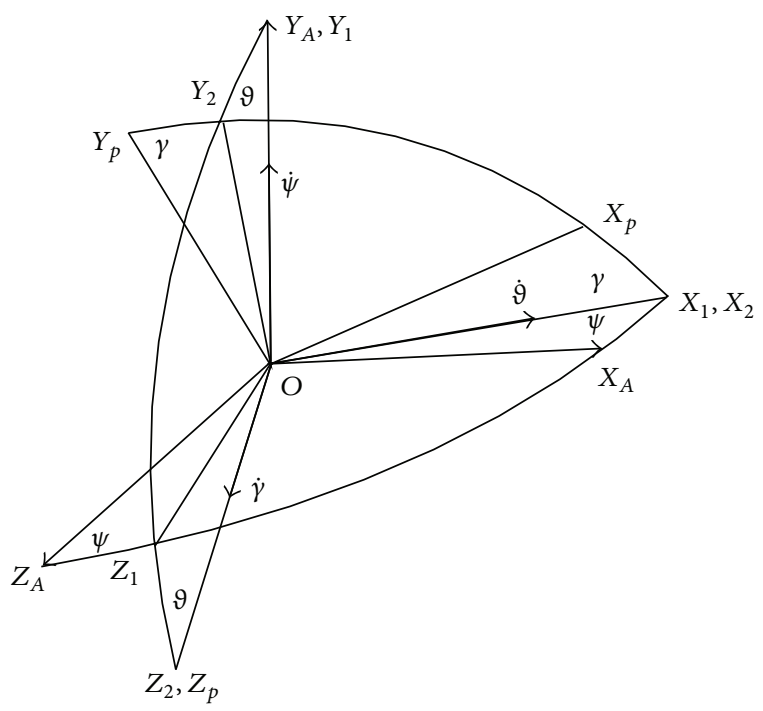

FIGURE 1: Sequence of rotations determining the platform position.

$$
\begin{aligned}
{\left[\begin{array}{c}
\omega_{X} \\
\omega_{Y} \\
\omega_{Z}
\end{array}\right]=} & A_{3}^{T} A_{2}^{T} A_{1}^{T}\left[\begin{array}{l}
\omega_{X_{A}} \\
\omega_{Y_{A}} \\
\omega_{Z_{A}}
\end{array}\right] \\
& +A_{3}^{T}\left(A_{2}^{T} A_{1}^{T}\left[\begin{array}{l}
0 \\
\dot{\psi} \\
0
\end{array}\right]+A_{2}^{T}\left[\begin{array}{l}
\dot{\vartheta} \\
0 \\
0
\end{array}\right]+\left[\begin{array}{l}
0 \\
0 \\
\dot{\gamma}
\end{array}\right]\right) \\
= & A^{T}\left[\begin{array}{c}
\omega_{X_{A}} \\
\omega_{Y_{A}} \\
\omega_{Z_{A}}
\end{array}\right]+\left[\begin{array}{c}
\dot{\vartheta} \cos \gamma+\dot{\psi} \cos \vartheta \sin \gamma \\
-\dot{\vartheta} \sin \gamma+\dot{\psi} \cos \vartheta \cos \gamma \\
\dot{\gamma}-\dot{\psi} \sin \vartheta
\end{array}\right] .
\end{aligned}
$$

The first components of the obtained formula (11) are the projections of UAV angular rate onto the platform axes. In other words, these constituents represent projections of the rate of translation. The second constituents define appropriate projections of the platform relative angular rate.

While creating the mathematical model of the stabilization system it is necessary to take into account that the control moments are formed relatively to the gimbals of the yaw, pitch, and roll $\psi, \vartheta, \gamma$ and the constituents of relationships (1) are projections onto the proper axes of the platform. Therefore the expressions for the control moments determination must be based on the following transformations:

$$
\begin{aligned}
& {\left[\begin{array}{l}
M_{X p} \\
M_{Y p} \\
M_{Z p}
\end{array}\right]=\mathbf{A}_{3}^{T}\left[\begin{array}{c}
0 \\
0 \\
M_{Z \gamma}
\end{array}\right]=\left[\begin{array}{c}
0 \\
0 \\
M_{Z \gamma}
\end{array}\right] ;} \\
& {\left[\begin{array}{l}
M_{X p} \\
M_{Y p} \\
M_{Z p}
\end{array}\right]=\mathbf{A}_{3}^{T} \mathbf{A}_{2}^{T}\left[\begin{array}{c}
M_{X \vartheta} \\
0 \\
0
\end{array}\right]=\left[\begin{array}{c}
\cos \gamma M_{X \vartheta} \\
-\sin \gamma M_{X \vartheta} \\
0
\end{array}\right] ;} \\
& {\left[\begin{array}{l}
M_{X p} \\
M_{Y p} \\
M_{Z p}
\end{array}\right]=\mathbf{A}_{3}^{T} \mathbf{A}_{2}^{T} \mathbf{A}_{1}^{T}\left[\begin{array}{c}
0 \\
M_{Y \psi} \\
0
\end{array}\right]=\left[\begin{array}{c}
\cos \vartheta \sin \gamma M_{Y \psi} \\
\cos \vartheta \cos \gamma M_{Y \psi} \\
-\sin \vartheta M_{Y \psi}
\end{array}\right] .}
\end{aligned}
$$

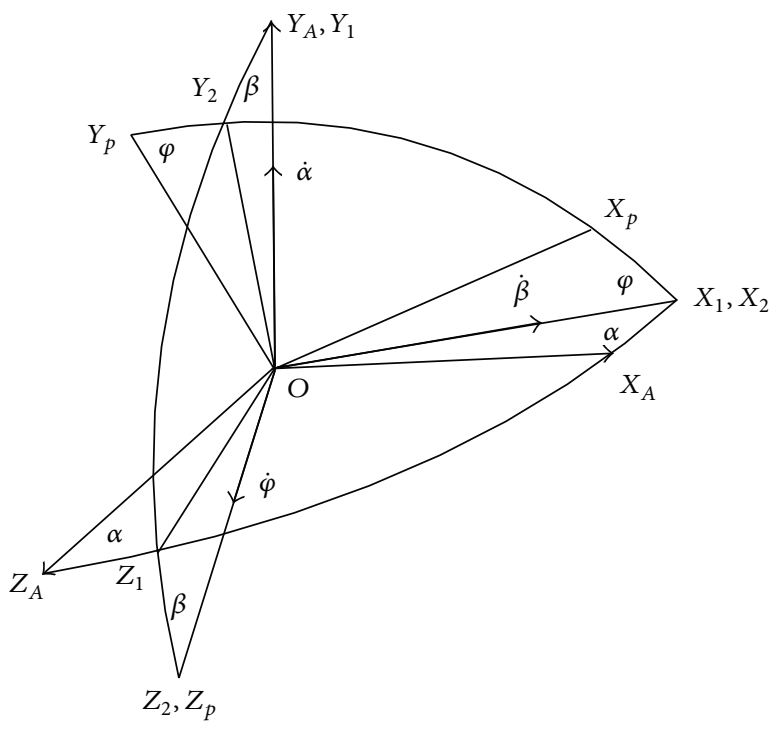

FIGURE 2: Sequence of rotations of the gimbals axes during platform stabilization.

Based on expressions (12) the projections of the moments on the platform axes are represented as

$$
\begin{aligned}
& M_{X p}=\cos \gamma M_{X \vartheta}+\cos \vartheta \sin \gamma M_{Y \psi} ; \\
& M_{Y p}=-\sin \gamma M_{X \vartheta}+\cos \vartheta \cos \gamma M_{Y \psi} ; \\
& M_{Z p}=M_{Z \psi}-\sin \vartheta M_{Y \psi} .
\end{aligned}
$$

It should be noticed that angular rates of the platform are defined by expressions (9)-(11) if the gimbals axes coincide with the UAV axes at the initial instant of time. Taking into consideration this supposition, the sequence of rotations of the platform with the installed equipment and measuring instruments during stabilization will correspond to the sequence of rotations shown in Figure 2.

In accordance with Figure 2 the vector of the stabilization rate can be determined by the expression

$$
\Omega=\dot{\alpha} Y_{p}+\dot{\beta} X_{1}+\dot{\varphi} Z_{2}
$$

where $\dot{\alpha}, \dot{\beta}, \dot{\varphi}$ are angular rates of the rotations of the external and internal gimbals.

Conditions of the precision stabilization (8) expressed in the projections on the platform axes can be represented in the following form:

$$
\begin{aligned}
& \Omega_{X}+\omega_{X}=0 ; \\
& \Omega_{Y}+\omega_{Y}=0 ; \\
& \Omega_{Z}+\omega_{Z}=0 .
\end{aligned}
$$

Projections of stabilization rates of the platform on proper axes in accordance with Figure 2 could be defined by the 
following relationships:

$$
\begin{aligned}
& \Omega_{X}=\dot{\beta} \cos \varphi+\dot{\alpha} \cos \beta \sin \varphi ; \\
& \Omega_{Y}=-\dot{\beta} \sin \varphi+\dot{\alpha} \cos \beta \cos \varphi ; \\
& \Omega_{Z}=\dot{\varphi}-\dot{\alpha} \sin \beta .
\end{aligned}
$$

Based on relationships (16) and taking into consideration (15) it is possible to derive expressions for angular rates of the platform stabilization

$$
\begin{aligned}
\dot{\alpha} & =-\frac{1}{\cos \beta}\left(\omega_{X} \sin \varphi+\omega_{Y} \cos \varphi\right) ; \\
-\omega_{Y} & =-\dot{\beta} \sin \varphi+\dot{\alpha} \cos \beta \cos \varphi ; \\
-\omega_{Z} & =\dot{\varphi}-\dot{\alpha} \sin \beta .
\end{aligned}
$$

After some transformations, using expressions (17), it is possible to obtain the differential equations for determination of the angular rates of the gimbals stabilization

$$
\begin{aligned}
& \dot{\alpha}=-\frac{1}{\cos \beta}\left(\omega_{X} \sin \varphi+\omega_{Y} \cos \varphi\right) ; \\
& \dot{\beta}=-\omega_{X} \cos \varphi+\omega_{Y} \sin \varphi ; \\
& \dot{\varphi}=\frac{\sin \beta}{\cos \beta}\left(\omega_{X} \sin \varphi+\omega_{Y} \cos \varphi\right)-\omega_{Z} .
\end{aligned}
$$

Relationships (1)-(5), (7), and (13) and (18) represent the mathematical model of the system for stabilization of the observation equipment operated on UAV.
In accordance with concepts of the modern control theory, design of robust systems is implemented in two stages. At the first stage the robust synthesis on the basis of the use of the linearized model in the state space is determined. At the second stage the check of the synthesized system by means of simulation is carried out. Depending on the obtained results the first stage may be implemented repeatedly after the change of the initial conditions and weighting transfer functions of the optimization criterion.

The robust optimization requires an application of the linearized model in the state space. Linearization of the derived model can be carried out using the following steps:

(1) neglecting the centrifugal moments and differences of axial moments of the platform that allows simplifying expressions (1),

(2) taking into consideration only small angles of the platform rotations which allows simplifying expressions (7), (13), and (18),

(3) linearization of nonlinear dry friction moments [17],

(4) using of the simplified linearized model of PWM,

(5) neglecting the disturbing moments acting on the platform.

Using expressions (1)-(6), (7), and (13) and (18) and the above listed steps the linear model of the stabilization plant for the vector of states $x$ can be described by the quadruple of the matrices $A, B, C, D$ in the state space

$$
\begin{aligned}
& x^{T}=\left[\begin{array}{lllllllllllllll}
\alpha & \beta & \gamma & U_{\omega X} & U_{\omega Y} & U_{\omega Z} & U_{a X} & U_{a Y} & U_{a Z} & \omega_{X} & \omega_{Y} & \omega_{Z} & U_{\omega d X} & U_{\omega d Y} & U_{\omega d Z}
\end{array}\right],
\end{aligned}
$$

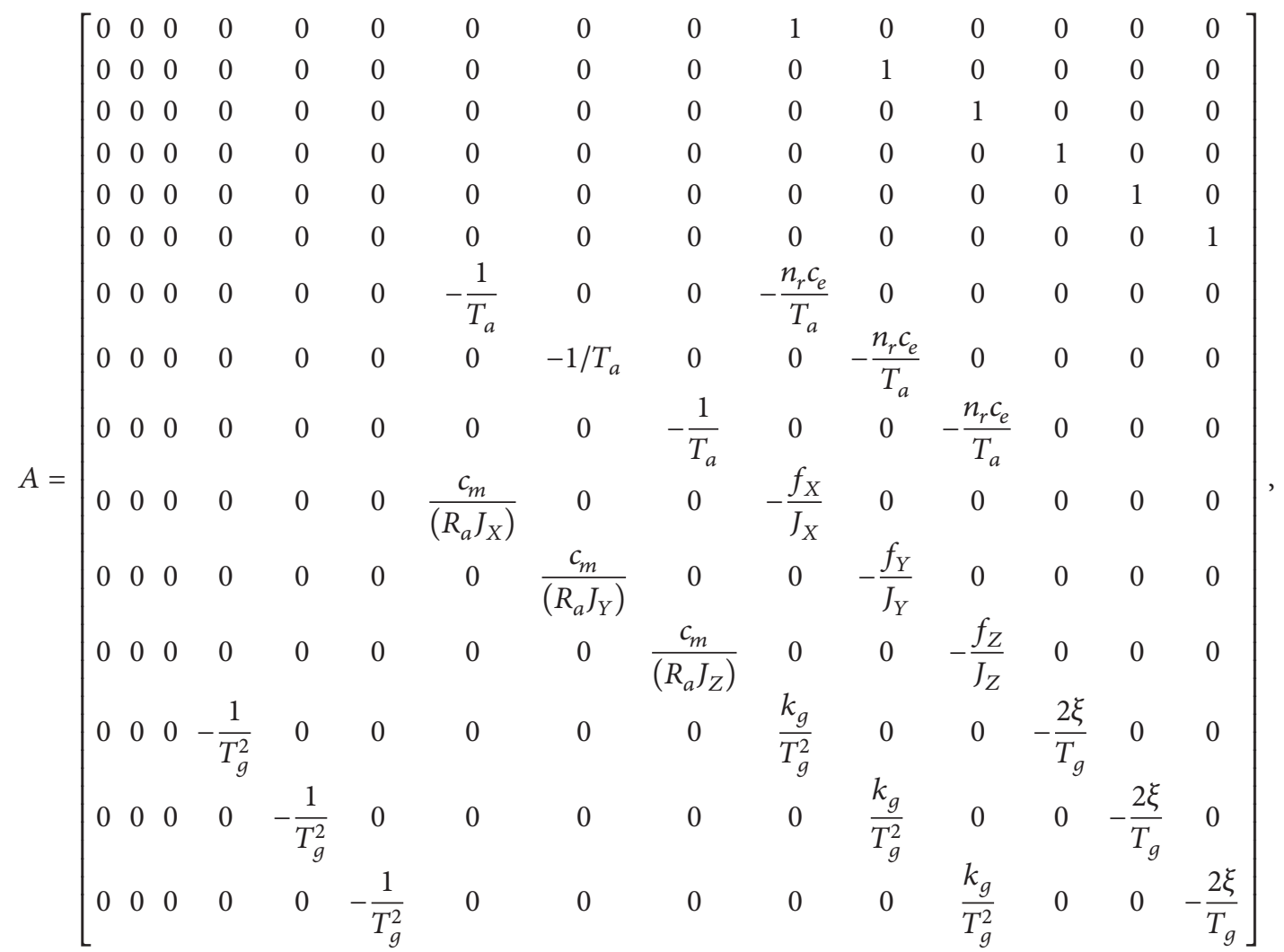




$$
\begin{aligned}
B^{T} & =\left[\begin{array}{llllllcccccccccc}
0 & 0 & 0 & 0 & 0 & 0 & k_{\mathrm{PWM}} & 0 & 0 & 0 & 0 & 0 & 0 & 0 & 0 \\
0 & 0 & 0 & 0 & 0 & 0 & 0 & k_{\mathrm{PWM}} & 0 & 0 & 0 & 0 & 0 & 0 & 0 \\
0 & 0 & 0 & 0 & 0 & 0 & 0 & 0 & k_{\mathrm{PWM}} & 0 & 0 & 0 & 0 & 0 & 0
\end{array}\right] \\
C & =\left[\begin{array}{lllllllllllllll}
0 & 0 & 0 & 1 & 0 & 0 & 0 & 0 & 0 & 0 & 0 & 0 & 0 & 0 & 0 \\
0 & 0 & 0 & 0 & 1 & 0 & 0 & 0 & 0 & 0 & 0 & 0 & 0 & 0 & 0 \\
0 & 0 & 0 & 0 & 0 & 1 & 0 & 0 & 0 & 0 & 0 & 0 & 0 & 0 & 0
\end{array}\right] ; \\
D & =\left[\begin{array}{lll}
0 & 0 & 0 \\
0 & 0 & 0 \\
0 & 0 & 0
\end{array}\right] .
\end{aligned}
$$

Elements $U_{\omega d X}, U_{\omega d Y}, U_{\omega d Z}$ of the matrix $A$ represent derivatives of the voltages $U_{\omega X}, U_{\omega Y}, U_{\omega Z} ; f_{X}, f_{Y}, f_{Z}$ represent coefficients of linearized moments of the dry friction [17].

The mathematical models (19) can be used in the procedure of the robust structural optimization.

\section{Algorithm of Structural Synthesis of Robust Stabilization System}

One of the modern approaches to the structural synthesis of the robust stabilization systems is $H_{\infty}$-synthesis. Its basic principles are represented in many papers and textbooks, for example, [7, 8]. The standard configuration of the system designed by means of the $H_{\infty}$-synthesis is shown in Figure 3.

The system consists of the plant $G$ and the controller $K$ and is characterized with the vectors of output parameters $z$ to be optimized, external input signals $w$, control signals $u$, and measured signals $y$ entering the input of the controller $[7,8]$.

The researched system can be described by the scheme represented in Figure 4 [8].

Interconnection between signals of such system can be described by the following expressions [8]:

$$
\begin{aligned}
& w=r \\
& z=\left[\begin{array}{l}
e \\
u \\
y
\end{array}\right] ; \\
& P=\left[\begin{array}{ll}
P_{11} & P_{12} \\
P_{21} & P_{22}
\end{array}\right]=\left[\begin{array}{cc}
I & -G \\
0 & I \\
0 & G \\
I & -G
\end{array}\right],
\end{aligned}
$$

where $P$ represents the transfer function of the interconnected system.
The transfer function from the input $w$ to the output $z$ becomes

$$
T_{w}^{z}=P_{11}+P_{12} K\left(I-P_{22} K\right)^{-1} P_{21} .
$$

The principle of the guaranteed result may be applied to robust systems. In accordance with this principle [7]

$$
\frac{\|P(s) w(s)\|_{\infty}}{\|w(s)\|_{\infty}}<\gamma,
$$

where $\gamma$ is a small value; $w(s)$ is representation of the external input signals including disturbances.

Optimization problem of the robust structural synthesis can be represented in the following form $[7,8]$ :

$$
K_{\mathrm{opt}}=\arg \inf _{K_{\mathrm{opt}} \in K_{\mathrm{per}}} J(G, K) \text {, }
$$

where

$$
J(G, K)=\left\|\left[\begin{array}{c}
(I+G K)^{-1} \\
K(I+G K)^{-1} \\
G K(I+G K)^{-1}
\end{array}\right]\right\|_{\infty}
$$

The optimization problem (23) may be solved by means of the method of the mixed sensitivity $[7,8]$. The modern approach to solving the problem of the robust structural optimization is based on forming the desired amplitudefrequency characteristics of the system (loop-shaping) by means of the plant augmentation due to introducing the weighting transfer functions as it is shown in Figure 5 [7].

Optimization criterion of the method of the mixed sensitivity can be represented based on formula (24) in the form of the expression representing the $H_{\infty}$-norm of the function of the mixed sensitivity of the augmented system $[7,8]$

$$
J(G, K)=\left\|\left[\begin{array}{c}
W_{1}(I+G K)^{-1} \\
W_{2} K(I+G K)^{-1} \\
W_{3} G K(I+G K)^{-1}
\end{array}\right]\right\|_{\infty}=\left\|\left[\begin{array}{c}
W_{1} S \\
W_{2} R \\
W_{3} T
\end{array}\right]\right\|_{\infty},
$$




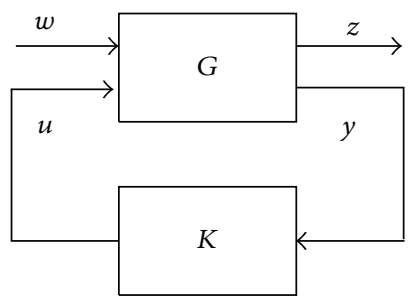

FIGURE 3: Standard $H_{\infty}$-configuration.

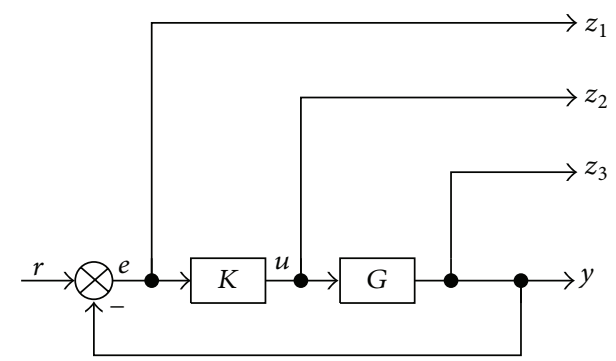

FIGURE 4: Structural scheme of the designed system.

where $W_{1}, W_{2}, W_{3}$ represent the weighting transfer functions; $S, R, T$ are functions of sensitivity with respect to the reference and control signals and complementary sensitivity function.

Implementation of the $H_{\infty}$-synthesis by the method of the mixed sensitivity is based on solutions of two Riccati equations, checking some conditions and minimization of the $H_{\infty}$-norm of the function of the mixed sensitivity $(25)[7,8]$. It should be noticed that there are automated means of this problem solution based on MatLab software. The appropriate optimization procedure requires using the mathematical model of the interconnected system (20). as

The procedure of the $H_{\infty}$-synthesis includes such stages

(1) creation of the mathematical models including the linear model and the model which takes into consideration the nonlinearities inherent to real systems,

(2) choice of the weighting transfer functions,

(3) augmentation of the plant,

(4) structural robust synthesis.

These stages can be implemented by means of Robust Control Toolbox of MatLab software.

One of the most important stages of the robust structural synthesis is a choice of the weighting transfer functions, which provide forming of the augmented plant and loopshaping approach. There are the following general recommendations for determination of weighting transfer functions for augmented plant forming [7]:

$$
\begin{aligned}
& W_{1}=\frac{s / M+\omega_{0}}{s+\omega_{0} A} ; \\
& W_{2}=\text { const; } \\
& W_{3}=\frac{s+\omega_{0} / M}{A s+\omega_{0}},
\end{aligned}
$$

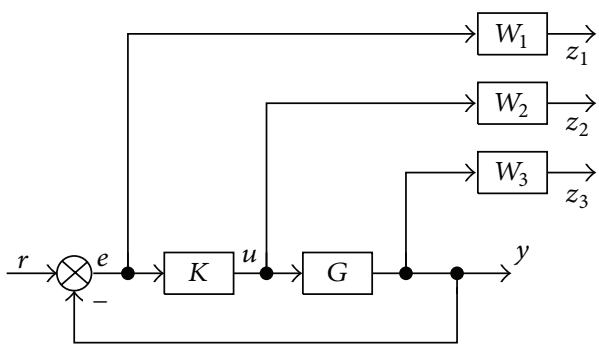

FIGURE 5: Structural scheme of the system augmented by means of the weighting transfer functions.

where $A$ is the desired stable error in the steady mode; $\omega_{0}$ is the desired bandwidth; $M$ is sensitivity peak.

An experience of the rate drives design showed the necessity of using integrator in the expression of the weighting transfer function $W_{1}$. But in real applications it is necessary to specify weighting transfer functions by methods of trials and errors.

In accordance with Figure 5 the weighting transfer functions impose a penalty on the error, control and output signals. Changing parameters of the weighting transfer functions $A, \omega_{0}, M$ it is possible to achieve the desired frequency characteristics of the system [7].

Usage of the weighting transfer functions provides loopshaping of the designed system. Such approach allows forming the designed system with the desired frequency characteristics.

During synthesis of the researched system the following expressions for the weighting transfer functions determination were obtained:

$$
\begin{aligned}
& W_{1}=\left[\begin{array}{ccc}
\frac{1}{s} \frac{0.1 s+20}{s+0.005} & 0 & 0 \\
0 & \frac{1}{s} \frac{0.02 s+10}{s+0.002} & 0 \\
0 & 0 & \frac{1}{s} \frac{0.01 s+10}{s+0.001}
\end{array}\right] ; \\
& W_{2}=\left[\begin{array}{ccc}
0.04 & 0 & 0 \\
0 & 0.04 & 0 \\
0 & 0 & 0.04
\end{array}\right] ; \\
& W_{3}=\left[\begin{array}{ccc}
\frac{s}{0.005 s+50} & 0 & 0 \\
0 & \frac{s}{0.002 s+20} & 0 \\
0 & 0 & \frac{s}{0.001 s+10}
\end{array}\right] .
\end{aligned}
$$

Synthesis of the robust controller was implemented by means of MatLab function hinfopt. First, an order of the synthesized controller was equalled to 8 . Decrease of controller order was implemented by means of function schmr. This allows decreasing the controller order to 5. Discretization of controller was implemented by means of function $c 2 d$.

Manufacturing experience showed that controller of such order is realizable in the form of digital controller. In this case 


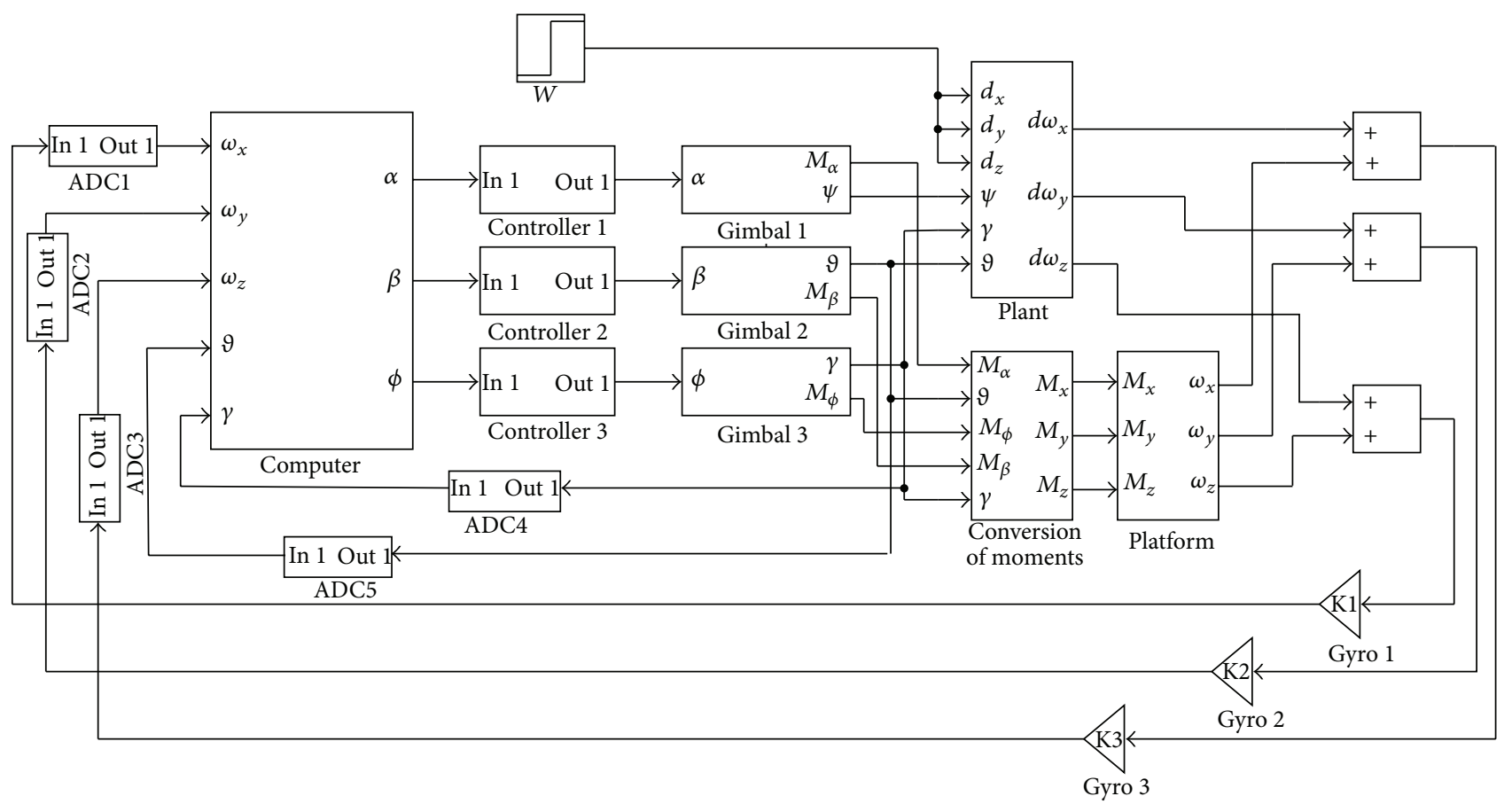

FIGURE 6: Simulink-model of the stabilization system.

the difference equations are realized by means of C-language assigned for microcontrollers.

The structure of the synthesized robust controller is described in the state space by means of the following quadruple of matrices:

$$
\begin{aligned}
& A_{p}=\left[\begin{array}{ccccc}
186.3 & 352.6 & 45.83 & 11.33 & 43.7 \\
711.8 & -966.3 & -179.7 & -12.17 & -11.6 \\
-117.75 & 126.7 & -18.41 & -15.84 & -15.62 \\
-343.7 & 517.3 & 136.8 & 19.39 & -23.43 \\
-218.3 & 268.8 & 20.53 & 18.75 & -25.89
\end{array}\right] \\
& B_{p}^{T}=\left[\begin{array}{ccccc}
20.98 & -37.1 & -8.3 & -5.36 & -7.25 \\
17.6 & -32.1 & -7.3 & -4.2 & 6.23 \\
15.6 & -30.5 & 5.4 & -3.1 & -5.21
\end{array}\right] \\
& C_{p}=\left[\begin{array}{ccccc}
8.31 & -9.8 & -4.78 & -1.43 & 2.35 \\
9.6 & -6.4 & 12.2 & -1.2 & 16.3 \\
11.2 & -7.5 & 9.2 & -11.1 & -15.21
\end{array}\right] \text {; } \\
& D_{p}=\left[\begin{array}{rrr}
-0.9 & -0.543 & -0.217 \\
-0.6 & -0.312 & -0.123 \\
-0.4 & -0.213 & -0.223
\end{array}\right] \text {. }
\end{aligned}
$$

The Simulink-model for system simulation is represented in Figure 6. Results of the synthesized system simulation are represented in Figures 7 and 8.

The angular rates of UAV, taking into consideration influence of the wind, were considered as disturbances during simulation. Forming the wind influence was carried out by means of the Dryden filter. Represented results prove the possibility of achieving the acceptable quality of the stabilization accuracy and speed of operation in conditions of influence of the external disturbances.

Advantages in comparison with other controllers may be described in the following way. Such systems keep required performances in conditions of changed parameters and external disturbances. Controllers of other types do not guarantee even stability of the system in such conditions. This can lead to inability to perform functions of the system not to mention providing of its high characteristics.

Adaptive systems take into consideration changes of plant characteristics during operation but they are too expensive in comparison with the robust systems.

\section{Conclusions}

(a) The kinematics expressions for three gimbal configurations were derived.

(b) The mathematical model of the stabilization plant of the considered system was obtained.

(c) The weighting transfer functions, which provide loop-shaping of the designed system, were determined.

(d) The robust controller was synthesized. Representation in the form of the quadruple of the state space matrices is convenient for digital controller programming.

(e) In order to provide the high accuracy of observation processes it is necessary to use the three-axial stabilization systems. 


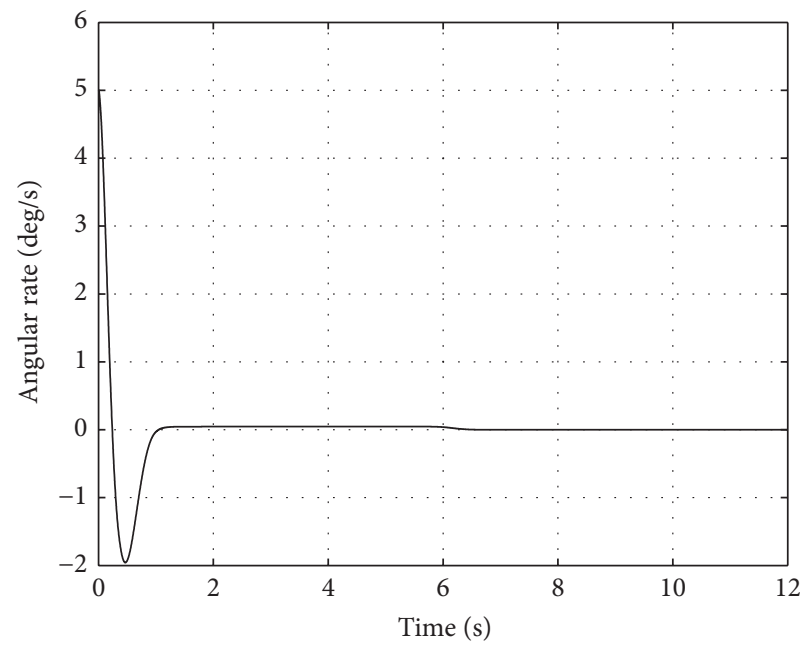

(a)

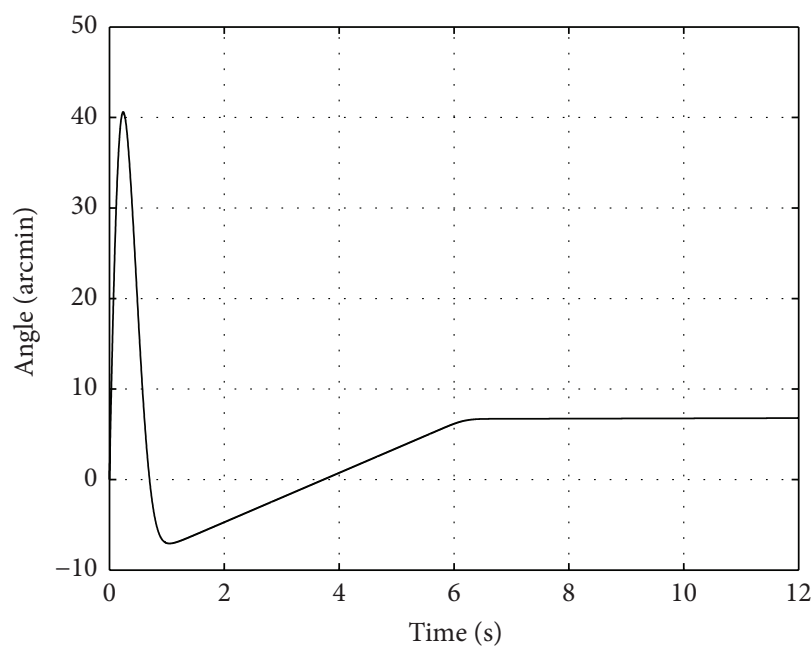

(b)

FiguRE 7: Results of simulation of the platform motion in the mode of prestabilization: (a) is transient of the given angular rate; (b) is error of the angular position determination.

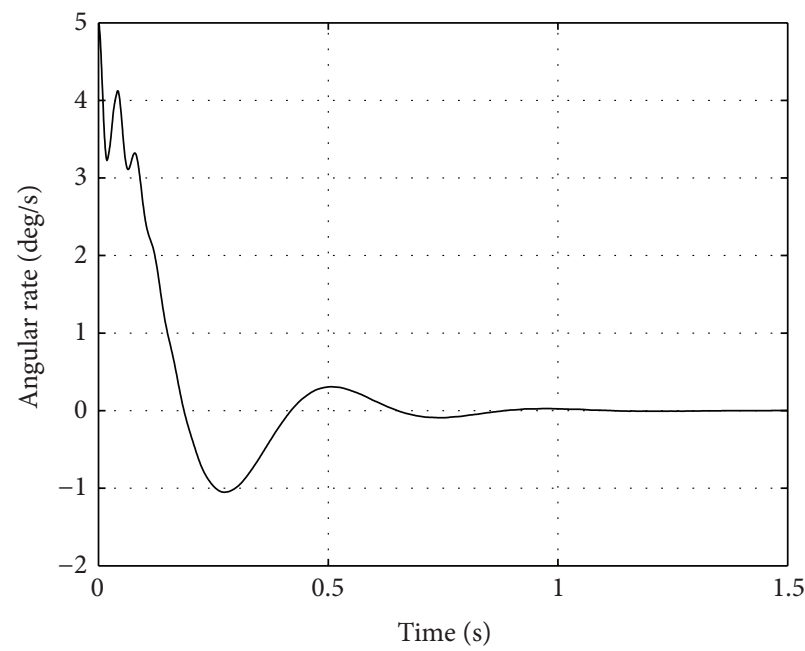

(a)

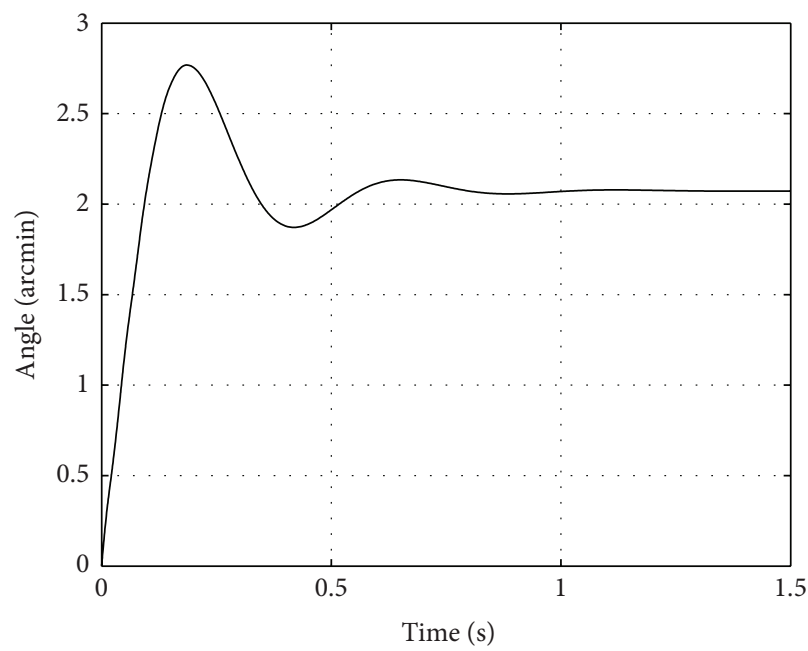

(b)

FIGURE 8: Results of simulation of the platform motion in the mode of precision stabilization: (a) is transient of the given angular rate; (b) is error of the angular position determination.

(f) Creation of the mathematical model of the three-axial stabilization system requires using the coordinate transformations.

(g) Efficiency of the suggested approaches is proved by the results of simulation of the synthesized system, while simulation influence of the wind was taken into consideration.

(h) The obtained results will be useful perspective for stabilization of the observation equipment operated on the vehicles of the wide class.

\section{Competing Interests}

The authors declare that there is no conflict of interests regarding the publication of this paper.

\section{References}

[1] J. M. Hilkert, "Inertially stabilized platform technology: concepts and principles," IEEE Control Systems Magazine, vol. 28, no. 1, pp. 26-46, 2008.

[2] M. K. Masten, "Inertially stabilized platforms for optical imaging systems," IEEE Control Systems Magazine, vol. 28, no. 1, pp. 47-64, 2008.

[3] D. Debruin, "Control systems for mobile satcom antennas," IEEE Control Systems Magazine, vol. 28, no. 1, pp. 86-101, 2008.

[4] H. G. Wang and T. C. Williams, "Strategic inertial navigation systems-high-accuracy inertially stabilized platforms for hostile environments," IEEE Control Systems Magazine, vol. 28, no. 1, pp. 65-85, 2008.

[5] B. A. Francis, J. W. Helton, and G. Zames, " $H^{\infty}$-optimal feedback controllers for linear multivariable systems," IEEE 
Transactions on Automatic Control, vol. 29, no. 10, pp. 888-900, 1984.

[6] K. Zhou and J. Doyle, Essentials of Robust Control, Prentice Hall, New Jersey, NJ, USA, 1999.

[7] S. Skogestad and I. Postlethwaite, Multivariable Feedback Control, John Wiley \& Sons, New York, NY, USA, 2001.

[8] D. W. Gu, P. H. Petkov, and M. M. Konstantinov, Robust Control Design with MATLAB, Springer, London, UK, 2005.

[9] A. A. Tunik, J. C. Kim, and C. S. Yoo, "The parameter optimization of aircrafts control law from the viewpoint of some airworthiness requirements," in Proceedings of the 12th Korea Automatic Control Conference (KACC'97), pp. 1651-1654, Seoul, South Korea, 1997.

[10] A. A. Tunik, H. Rye, and H. C. Lee, "Parametric optimization procedure for robust flight control system design," KSAS International Journal, vol. 2, no. 2, pp. 95-107, 2001.

[11] O. A. Sushchenko and A. A. Tunik, "Robust stabilization of UAV observation equipment," in Proceedings of the IEEE 2nd International Conference on Actual Problems of Unmanned Air Vehicles Developments (APUAVD '13), pp. 176-180, IEEE, Kiev, Ukraine, October 2013.

[12] O. A. Sushchenko, "Approach to design of robust systems for stabilization of unmanned aerial vehicles equipment," in Proceedings of the 6th World Congress 'Aviation in XX-st Century' 'Safety in Aviation and Space Technologies', vol. 2, pp. 3.5.1-3.5.15, Kyiv, Ukraine, September 2014.

[13] A. V. Goncharenko, "Expediency of unmanned air vehicles application in the framework of subjective analysis," in Proceedings of the IEEE 2nd International Conference on Actual Problems of Unmanned Air Vehicles Developments (APUAVD '13), pp. 129-133, IEEE, Kyiv, Ukraine, October 2013.

[14] M. A. Aizerman, Classical Mechanics, Nauka, Moscow, Russia, 1980 (Russian).

[15] O. A. Sushchenko, "Mathematical model of inertially stabilized platform for aircraft observation equipment," in Proceedings of the 4th World Congress Aviation in the 21st Century. Safety in Aviation and Space Technology, vol. 1, pp. 21.43-21.46, Kyiv, Ukraine, September 2010.

[16] D. S. Pelpor, Gyroscopic Systems, part 1, Vishaya Shkola, Moscow, Russia, 1976 (Russian).

[17] V. V. Kochergin, Servo-Systems with Direct Current Motors, Ehergoatomizdat, St-Petersburg, Russia, 1988 (Russian).

[18] S. S. Rivkin, Stabilization of Information and Measuring Devices on Swinging Base, Nauka, Moscow, Russia, 1978 (Russian). 


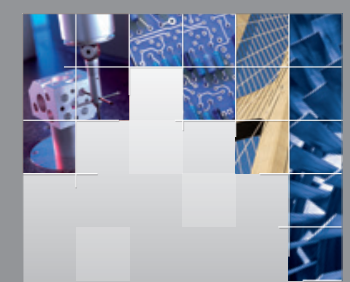

\section{Enfincering}
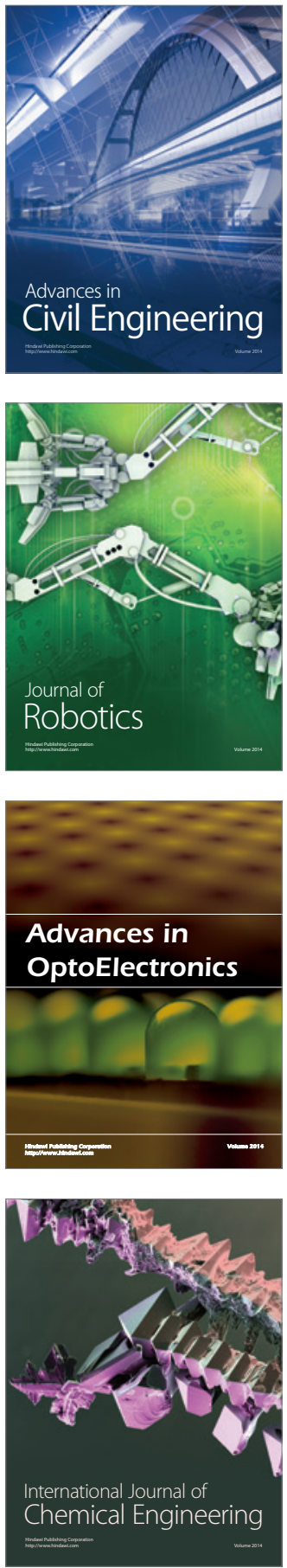

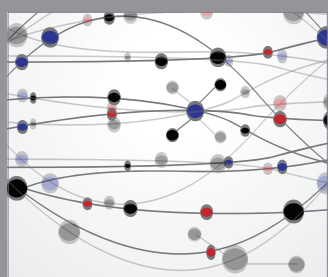

The Scientific World Journal

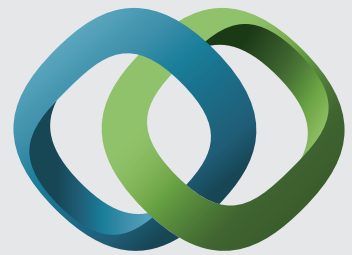

\section{Hindawi}

Submit your manuscripts at

http://www.hindawi.com
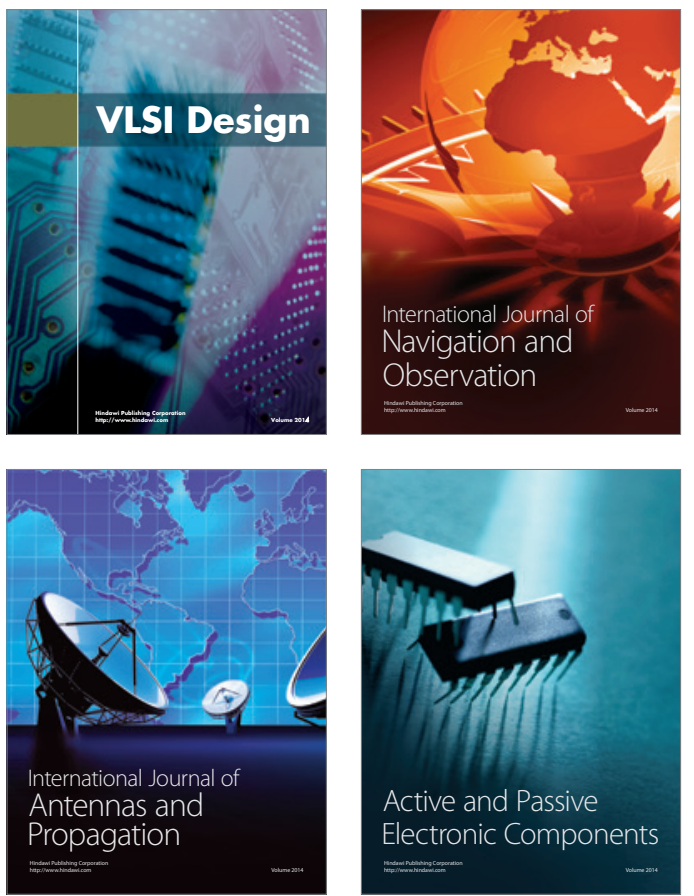
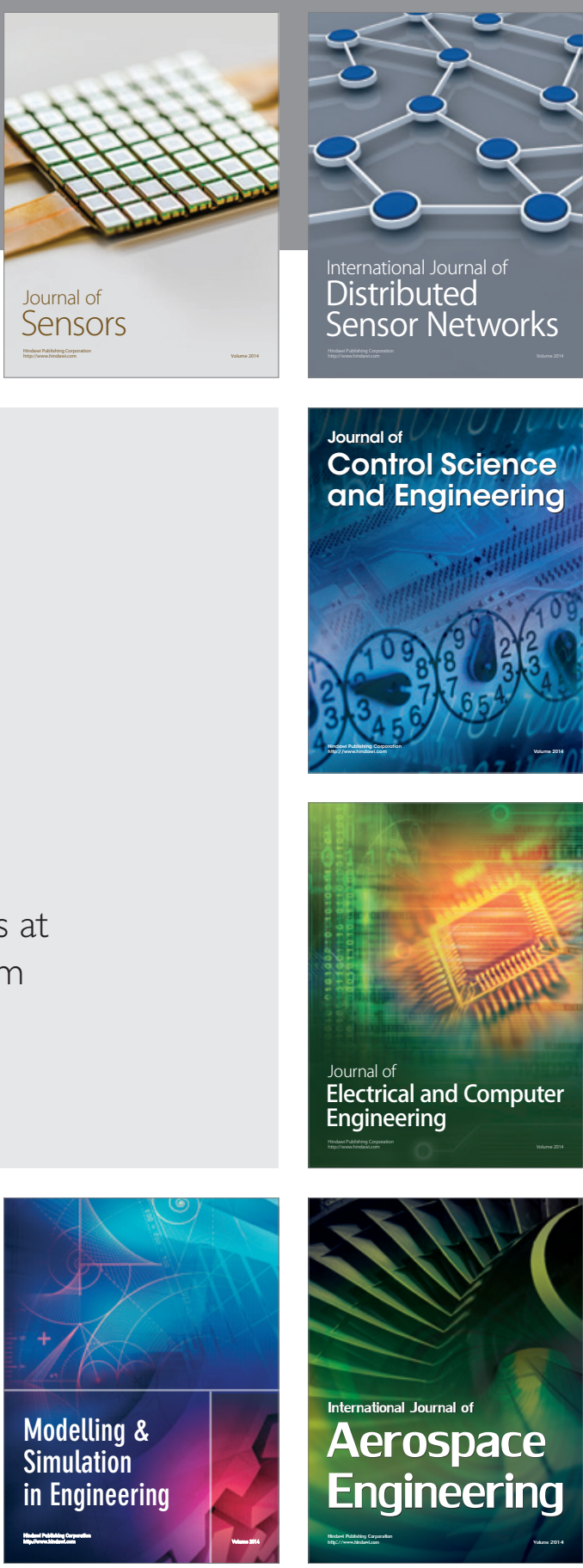

International Journal of

Distributed

Sensor Networks

Journal of

Control Science

and Engineering
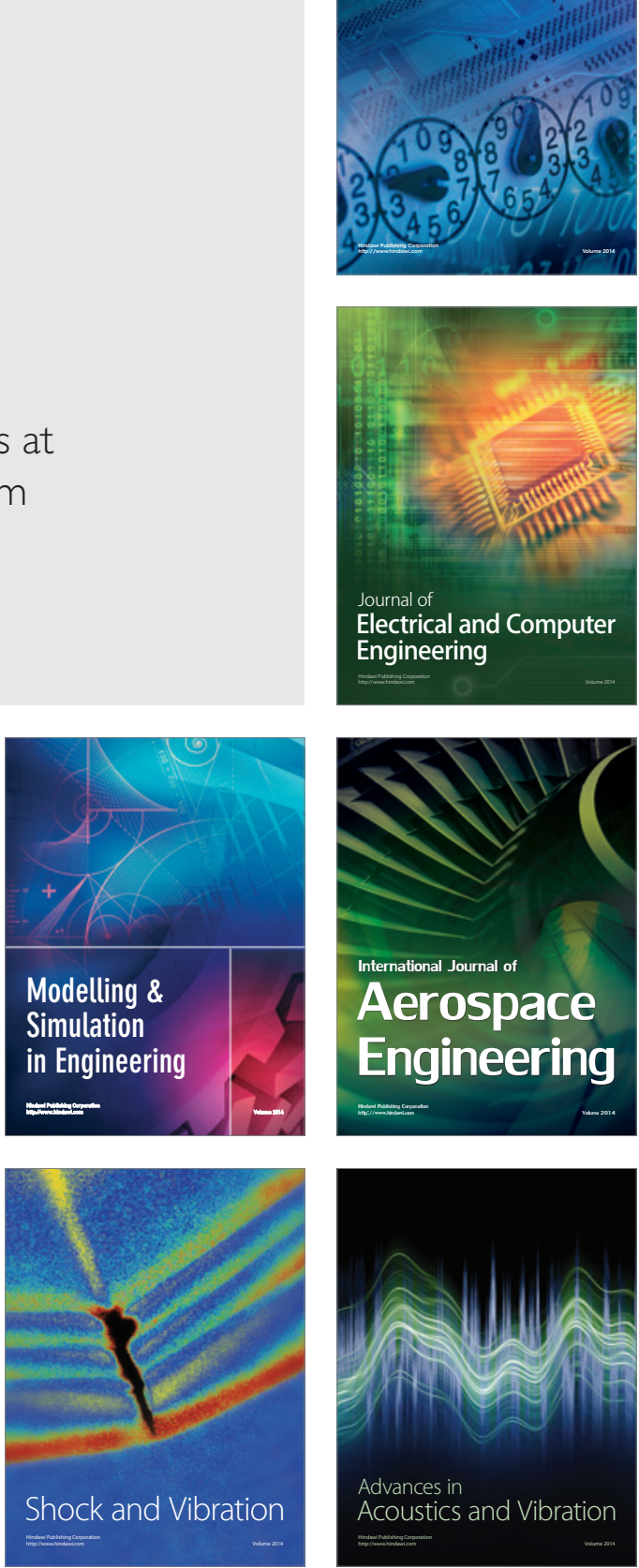